(c) 2016 IEEE. Personal use of this material is permitted. Permission from IEEE must be obtained for all other uses, in any current or future media, including reprinting/republishing this material for advertising or promotional purposes, creating new collective works, for resale or redistribution to servers or lists, or reuse of any copyrighted component of this work in other works 


\title{
Assessment of Sedation-Analgesia by means of Poincaré Analysis of the Electroencephalogram*
}

\author{
José D. Bolaños, Montserrat Vallverdú, Pere Caminal, Daniel F. Valencia, Xavi Borrat, Pedro L. \\ Gambús, and José F. Valencia, Member, IEEE
}

\begin{abstract}
Monitoring the levels of sedation-analgesia may be helpful for managing patient stress on minimally invasive medical procedures. Monitors based on EEG analysis and designed to assess general anesthesia cannot distinguish reliably between a light and deep sedation. In this work, the Poincaré plot is used as a nonlinear technique applied to EEG signals in order to characterize the levels of sedation-analgesia, according to observed categorical responses that were evaluated by means of Ramsay Sedation Scale (RSS). To study the effect of high frequencies due to EMG activity, three different frequency ranges $(F R 1=0.5-110 \mathrm{~Hz}, F R 2=0.5-30 \mathrm{~Hz}$ and $F R 3=30-110 \mathrm{~Hz})$ were considered. Indexes from power spectral analysis and plasma concentration of propofol and remifentanil were also compared with the bispectral index BIS. An adaptive Neurofuzzy Inference System was applied to model the interaction of the best indexes with respect to RSS score for each analysis, and leave-one-out cross validation method was used. The ability of the indexes to describe the level of sedation-analgesia, according with the RSS score, was evaluated using the prediction probability $(\mathrm{Pk})$. The results showed that the ratio SD1/SD2Fr3 contains useful information about the sedation level, and $\mathrm{SD1} 1_{\mathrm{FR} 2}$ and $\mathrm{SD2} 2_{\mathrm{FR} 2}$ had the best performance classifying response to noxious stimuli. Models including parameters from Poincaré plot emerge as a good estimator of sedation-analgesia levels.
\end{abstract}

\section{INTRODUCTION}

Adequate sedation and analgesia may be helpful for managing patient stress on minimally invasive medical procedures as endoscopies. Monitoring the levels of sedation-analgesia may provide appropriate requirements to control the administration of sedative drugs, minimizing the impact on aggression and on outcome process in the patient. Various anesthesia monitors have been introduced into clinical practice [1]-[3], providing different

* Research supported by COLCIENCIAS (1217-669-45428 / 297-2015) from Colombia government; MINECO (TEC2013-44666-R and TEC2014-603337-R) from Spanish Government. CIBER of Bioengineering, Biomaterials and Nanomedicine is an initiative of ISCIII. The End of Residency Award of Hospital CLINIC de Barcelona and the FIS (Fondo de Investigaciones Sanitarias, Health Department, Government of Spain) grants $\mathrm{n}^{\circ} \mathrm{PI} / 050072$ and PS09/01209.

J. D. Bolaños, D. F. Valencia and J. F. Valencia are with the Dept. of Electronic Engineering, Univ. San Buenaventura, Cali, Colombia (jdbolanos / dfvalencia1 / jfvalenc / @ usbcali.edu.co).

M. Vallverdú is with the ESAII Dept., Research Center CREB in Barcelona Tech, CIBER of Bioengineering, Biomaterials and Nanomedicine (CIBER-BBN), Spain (montserrat.vallverdu / pere.caminal / @ upc.edu).

X. Borrat and P. L. Gambús are with the SPEC-M Research Group. Dept. of Anesthesia, Hospital CLINIC de Barcelona, Spain (xborrat / plgambus / @ hospitalclinic.org). electroencephalogram (EEG) indexes to predict loss of consciousness, such as bispectral (BIS) index, auditory-evoked potential (AEP) index and cerebral state index (CSI). Nevertheless, these monitoring systems may offer inadequate anesthesia detection due to different reasons [4]-[6], such as due to the time varying dynamics involved in the EEG cerebral function that is very sensitive to the state of the patient. Therefore, monitors designed to assess deep levels of sedation as in general anesthesia cannot distinguish reliably between a light and deep sedation [7].

Although recently studies based on nonlinear signal processing dynamics in the brain could determine certain associated changes between EEG complexities [8] and the prediction of the painful stimulation in the conscious and unconscious state induced by anesthesia, these EEG changes are not fully understood. This suggests to extent the analysis of EEG signals to more methods in the time-domain and spectral-domain analysis, in order to find robust predictors of the level of sedation-analgesia. An interesting and simple nonlinear method is the Poincaré plot, which can analyze the variability of time series by describing the behavior of the signal in a phase-space trajectory as a function of a constant time delay. Some published works [9],[10] state that it is a valuable method due to its ability to exhibit nonlinear features in the time domain series, and it can be used in the analysis of nonfiltered and also nonstationary data. Therefore, it can be a useful method to explain complex brain dynamic regulatory processes during anesthesia, sleep and consciousness.

In this work, the Poincaré plot is used to characterize the levels of sedation-analgesia by analyzing EEG signals. Because the slow response to painful stimulation still remains an open problem, the prediction of these responses using the proposed indexes is going to be quantified. Indexes from power spectral analysis and plasma concentration of propofol and remifentanil are also compared with the bispectral index BIS.

\section{METHODOLOGY}

\section{A. Data Base}

Data were recorded, after receiving approval from the Ethics Committee of Hospital Clinic de Barcelona and signed informed consent, from 110 patients scheduled to undergo ultrasonographic endoscopy (USE) under sedation-analgesia. USE is a relatively long procedure with periods of stability of effect, allowing study of the repercussion of painful stimulus on the level of sedation. Every patient was routinely monitored, including: effect-site predicted concentrations of propofol (Cepro) and remifentanil (Ceremi) from a target 
controlled infusion system (FreseniusVial, Chemin de Fer, Béziers, France). The raw EEG signal was recorded with a sampling frequency of $900 \mathrm{~Hz}$, resolution of 16 bits and a recording time of about $60 \mathrm{~min}$ (acquired with the AEP monitor/2 - Danmeter, Odense, Denmark); and BIS of the EEG (continuously measured with an A2000 monitor - Aspect Medical Systems, Newton, MA). Observed categorical responses were evaluated by means of Ramsay Sedation Scale (RSS) [11]. RSS score (Table I) was estimated at random times during the procedure in order to avoid factors correlated with time, which could confound the results of the RSS measurements. In this study, the whole database contains annotated RSS scores from 2 to 6.

TABLE I.

THE RAMSAY SEDATION SCALE

\begin{tabular}{|c|l|}
\hline Score & Description \\
\hline 1 & Patient awake, anxious, agitated or restless \\
\hline 2 & Patient awake, cooperative, orientated and tranquil \\
\hline 3 & Patient drowsy with response to commands \\
\hline 4 & $\begin{array}{l}\text { Patient asleep, brisk response to glabella tap or loud auditory } \\
\text { stimulus }\end{array}$ \\
\hline 5 & Patient asleep, sluggish response to stimulus \\
\hline 6 & No response to firm nail-bed pressure or other noxious stimuli. \\
\hline
\end{tabular}

\section{B. Preprocessing}

EEG signals were resampled at $256 \mathrm{~Hz}$ after applying a Chebyshev low pass filter of $6^{\text {th }}$ order with cut-off frequency of $127 \mathrm{~Hz}$. Then, the EEG signals were segmented into windows of 1 minute length between $90 \mathrm{~s}$ and $30 \mathrm{~s}$ before the response annotation of RSS. Assuming that, in an ideal situation, the sedation level should be constant if plasma concentration of the anesthetic and analgesic agents remains without changes, the annotated RSS was assigned to the previous 1-minute length window if the differences in the predicted concentrations of remifentanil $(\Delta \mathrm{CeRemi})$ and propofol ( $\Delta$ CeProp), calculated between the first and the last second of the window, were $\Delta$ CeRemi $<0.1 \mathrm{ng} / \mathrm{ml}$ and $\Delta$ CeProp $<0.1 \mu \mathrm{g} / \mathrm{ml}$. Otherwise, the window was cut at the sample where the conditions were satisfied. EEG amplitudes out of the range of $+/-200 \mu \mathrm{V}$ were considered as artifact.

\section{Poincare Plot}

Poincare plot or return map is a phase space where a time series is represented in a Cartesian plane [12]. A phase space allows studying variations in a signal only with respect to itself. Given a time series $x(i), i=1, \ldots, N$, the Poincaré plot is constructed plotting $x(i)$ against $x(i+l a g)$, where the parameter lag is the time delay between $x$ and $y$ coordinates of the phase space (Fig. 1). Two standard descriptors used in quantifying the plot are $S D 1$ and $S D 2$, which can be obtain by fitting an ellipse to the plot shape as shown in Fig. 1. In terms of linear statistics, $S D 1$ measures the standard deviation of the points perpendicular to the line of identity and determines the width of the ellipse (short-term variability), whereas SD2 measures the standard deviation along the line of identity and determines the length of the ellipse (long-term variability) [10]. The $S D 1 / S D 2$ ratio characterizes the sharpness of the scatter pattern. According with [10] a lag of $8 \mathrm{~ms}$ was fixed in this work.

Epochs of 10 seconds were considered in the Poincaré analysis, with an overlapping of $90 \%$ between consecutive epochs, allowing $S D 1, S D 2$ and $S D 1 / S D 2$ ratio to be obtained each second. Also, to study the effect of high frequencies due to EMG signal content, the Poincaré analysis was applied to the time series in three different frequency ranges: $\mathrm{FR} 1=0.5-110 \mathrm{~Hz}, \mathrm{FR} 2=0.5-30 \mathrm{~Hz}$ (low frequency range) and FR3 $=30-110 \mathrm{~Hz}$ (high frequency range). Although EEG and EMG overlap their frequency range, FR2 emphasizes the EEG components and FR3 the EMG components.

Figure 1. Poincaré plot and descriptors SD1 and SD2

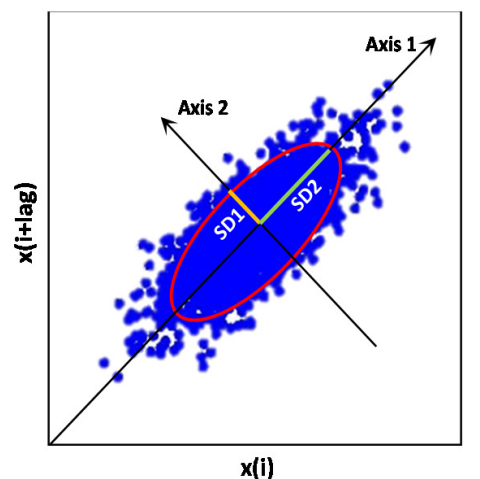

\section{Power Spectral Analysis}

The same epochs considered in the Poincaré analysis were also used for power spectral analysis. The power spectral density (PSD) for each EEG epoch was calculated using the FFT after the application of a Hamming window. Then, spectral power in each band $\left(P_{\delta}, 0.1-4 \mathrm{~Hz} ; P_{\theta}, 4-8 \mathrm{~Hz} ; P_{\alpha}, 8-\right.$ $\left.12 \mathrm{~Hz} ; P_{\beta}, 12-30 \mathrm{~Hz}\right)$ was computed as the area under the normalized PSD curve for the given frequency range. Spectral edge frequency 95\% (SEF95) and spectral entropy $(S p E n)$ were also calculated. SEF95 represents the frequency below which $95 \%$ of the power in the spectrum resides. This value decreases during induction of anesthesia as the power in high frequencies shifts toward lower frequencies [13]. SpEn quantifies the irregularity or complexity in the EEG. This value is low when the signal frequency components are concentrated on a small set of the spectrum, and increases the more uniformly the frequency components spread to all possible values of the spectrum [13].

\section{E. Statistics}

The ability of the indexes to describe the level of sedation-analgesia, according with the RSS score, was evaluated using the prediction probability $(\mathrm{Pk})$, which compares the performance of indicators [14]. The Pk coefficient is a statistic commonly used to measure how well an index predicts the state of the patient. A Pk of 1 represents a perfect prediction and 0.5 is not better than tossing a fair coin. Kruskal-Wallis test was applied in order to find statistically significant differences between RSS groups and the significance level was set at $\mathrm{p}$-value $<0.01$. Two analysis were considered: i) Trial 1, taking into account all the different RSS groups (RSS2, RSS3, RSS4, RSS5 and RSS6); ii) Trial 2, only considering groups RSS5 and RSS6, which correspond to the observed categorical responses after nail bed compression (noxious stimuli). Adaptive Neurofuzzy Inference System (ANFIS) was applied to model the interaction of the best 
indexes with respect to RSS score for each analysis, and leave-one-out cross validation method was used. The model was fit to the data by iteratively minimizing the root mean square errors using a backpropagation gradient descent method. In Trail 2, accuracy (Acc) was also computed.

\section{RESULTS}

\section{A. Assessment the level of sedation}

Table II shows the mean and the standard deviation, for each RSS of the indexes proposed in this work for Trial 1.
Also, the Pk computed considering all the scales is presented. Almost all the indexes showed a significant p-value between consecutive RSS groups, with the exception of: $P_{\alpha}$ (RSS5 vs RSS6), $S D 1_{F R 2}$ (RSS2 vs RSS3), and the indexes $S D 1_{F R 1}$, $S D 1_{F R 3}$ and $S D 2_{F R 3}$ in (RSS4 vs RSS5). Only three indexes $\left(B I S, S D 1_{F R 3}\right.$ and $\left.S D 1 / S D 2_{F R 3}\right)$ have a monotonic increase or decrease of the mean value as function of the RSS, though the standard deviation of $S D 1_{F R 3}$ was relatively very high, which is reflected in a low $\mathrm{Pk}$ value. The indexes with a $\mathrm{Pk} \geq 0.70$ were: $B I S, C e P r o p o, P_{\alpha}$ and $S D 1 / S D 2_{F R 3}$.

STATISTICAL PARAMETERS FOR EACH RAMSAY SEDATION SCALE: FR1=0.5-110 HZ, FR2=0.5-30 HZ AND FR3=30-110 HZ

\begin{tabular}{|c|c|c|c|c|c|c|}
\hline Index & $\begin{array}{c}\text { RSS2 } \\
\text { mean } \pm \text { std }\end{array}$ & $\begin{array}{c}\text { RSS3 } \\
\text { mean } \pm \text { std }\end{array}$ & $\begin{array}{c}\text { RSS4 } \\
\text { mean } \pm \text { std }\end{array}$ & $\begin{array}{c}\text { RSS5 } \\
\text { mean } \pm \text { std }\end{array}$ & $\begin{array}{c}\text { RSS6 } \\
\text { mean } \pm \text { std }\end{array}$ & Pk \\
\hline$B I S$ & $93.0 \pm 7.6$ & $82.0 \pm 10.8$ & $74.0 \pm 12.0$ & $65.6 \pm 14.2$ & $64.4 \pm 14.6$ & 0.778 \\
\hline$C e R e m i$ & $0.557 \pm 0.880$ & $1.10 \pm 0.827$ & $1.25 \pm 0.824$ & $0.967 \pm 0.876$ & $1.45 \pm 0.728$ & 0.614 \\
\hline CePropo & $0.527 \pm 0.792$ & $1.90 \pm 0.712$ & $2.16 \pm 0.670$ & $2.39 \pm 0.648$ & $2.19 \pm 0.628$ & 0.716 \\
\hline$P_{\delta}$ & $0.542 \pm 0.224$ & $0.295 \pm 0.210$ & $0.245 \pm 0.170$ & $0.230 \pm 0.171$ & $0.281 \pm 0.168$ & 0.625 \\
\hline$P_{\alpha}$ & $0.084 \pm 0.070$ & $0.177 \pm 0.109$ & $0.260 \pm 0.136$ & $0.317 \pm 0.141$ & $0.317 \pm 0.144$ & 0.755 \\
\hline$P_{\beta}$ & $0.201 \pm 0.212$ & $0.390 \pm 0.207$ & $0.342 \pm 0.173$ & $0.288 \pm 0.154$ & $0.216 \pm 0.128$ & 0.509 \\
\hline$S E F 95$ & $18.2 \pm 7.32$ & $22.5 \pm 4.34$ & $20.5 \pm 3.70$ & $18.9 \pm 3.12$ & $18.2 \pm 2.92$ & 0.588 \\
\hline$S p E n$ & $0.776 \pm 0.082$ & $0.846 \pm 0.062$ & $0.839 \pm 0.049$ & $0.826 \pm 0.048$ & $0.818 \pm 0.040$ & 0.501 \\
\hline$S D 1_{F R 1}$ & $10.8 \pm 7.25$ & $9.60 \pm 6.41$ & $8.93 \pm 5.13$ & $9.02 \pm 5.51$ & $8.14 \pm 5.55$ & 0.553 \\
\hline$S D 2_{F R 1}$ & $22.4 \pm 12.0$ & $15.6 \pm 7.27$ & $16.0 \pm 6.67$ & $17.2 \pm 7.05$ & $14.6 \pm 7.32$ & 0.576 \\
\hline$S D 1 / S D 2_{F R 1}$ & $0.526 \pm 0.282$ & $0.605 \pm 0.233$ & $0.550 \pm 0.195$ & $0.504 \pm 0.169$ & $0.531 \pm 0.169$ & 0.505 \\
\hline$S D 1_{\mathrm{FR} 2}$ & $3.22 \pm 1.34$ & $3.22 \pm 1.34$ & $3.27 \pm 1.36$ & $3.48 \pm 1.34$ & $2.55 \pm 1.32$ & 0.550 \\
\hline$S D 2_{F R 2}$ & $20.1 \pm 12.2$ & $12.1 \pm 6.06$ & $12.4 \pm 6.10$ & $13.6 \pm 5.90$ & $10.6 \pm 5.53$ & 0.606 \\
\hline$S D 1 / S D 2_{F R 2}$ & $0.193 \pm 0.091$ & $0.283 \pm 0.079$ & $0.273 \pm 0.057$ & $0.259 \pm 0.047$ & $0.241 \pm 0.043$ & 0.532 \\
\hline$S D 1_{F R 3}$ & $9.88 \pm 7.26$ & $8.60 \pm 6.61$ & $7.85 \pm 5.48$ & $7.82 \pm 5.98$ & $7.42 \pm 5.77$ & 0.553 \\
\hline$S D 2_{F R 3}$ & $7.21 \pm 5.69$ & $8.00 \pm 6.51$ & $8.34 \pm 6.04$ & $8.74 \pm 7.02$ & $8.47 \pm 7.04$ & 0.520 \\
\hline$S D 1 / S D 2_{F R 3}$ & $1.45 \pm 0.35$ & $1.18 \pm 0.34$ & $1.01 \pm 0.26$ & $0.981 \pm 0.244$ & $0.966 \pm 0.252$ & 0.711 \\
\hline
\end{tabular}

Indexes CePropo, $P_{\alpha}$ and $S D 1 / S D 2_{F R 3}$ were used as inputs of an ANFIS system (implemented in MATLAB) in order to model the RSS score, achieving a $\mathrm{Pk}=0.791$. Figure 2 shows the course of the ANFIS's output and the observed categorical responses of one EEG record.

Figure 2. Course of the ANFIS's output (blue line) and the observed categorical responses according to the RSS (red line).

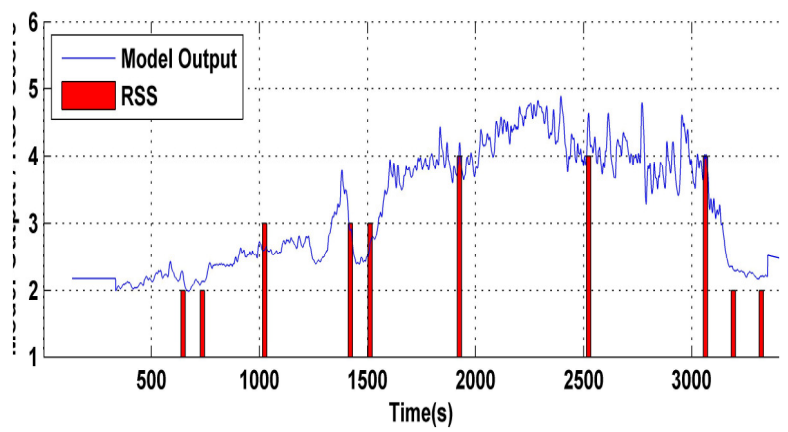

\section{B. Assessment the level of analgesia (nociception)}

Table III includes the indexes with $\mathrm{Pk}>=0.60$ for Trial 2 , when the observed categorical responses were classified in RSS5 and RSS6 after the application of nail bed painful stimulation, which is a kind of noxious stimuli. Although the indexes BIS, SEF95 and SpEn have $\mathrm{Pk}<0.55$, they were also included as reference. The individual index with the highest Pk (0.714) was $S D 1_{F R 2}$, while the ANFIS model showed the best $\mathrm{Pk}(0.780)$. The ANFIS system was trained using 4 inputs (CeRemi, $P_{\beta}, S D 1_{F R 2}$ and $S D 1_{F R 2}$ ) in order to estimate the scales RSS5 and RSS6, as a model to predict the response to noxious stimuli. The highest Acc $(70.9 \%)$ was also obtained with the output of the ANFIS model.

TABLE III. STATISTICS USING RSS=5 AND RSS=6 AS REFERENCE

\begin{tabular}{|c|c|c|c|c|c|c|c|c|}
\hline Index & BIS & CeRemi & $\boldsymbol{P}_{\boldsymbol{\beta}}$ & SEF95 & SpEn & $\boldsymbol{S D 1}_{F R 2}$ & $\boldsymbol{S D 2}_{F R 2}$ & ANFIS \\
\hline $\mathbf{P k}$ & $\begin{array}{c}0.52 \\
8\end{array}$ & 0.675 & 0.647 & 0.544 & 0.570 & 0.714 & 0.699 & 0.780 \\
\hline Acc (\%) & 52.6 & 63.1 & 61.6 & 53.4 & 54.8 & 67.1 & 67.1 & 70.9 \\
\hline
\end{tabular}

\section{Discussion}

In this work, Poincaré analysis was applied to EEG signals in three different frequency ranges: $F R 1=0.5-110 \mathrm{~Hz}$, FR2 $=0.5-30 \mathrm{~Hz}$ and FR3 $=30-110 \mathrm{~Hz}$, where FR2 emphasizes the EEG components and FR3 the EMG components. The assessment of the sedation, according with the RSS scores (Table II), showed that the ratio $S D 1 / S D 2_{F R 3}$, which was computed in the frequency range between $30-110 \mathrm{~Hz}$, contains useful information about the sedation level. A significant decrease in the $S D 1 / S D 2_{F R 3}$ ratio was observed with each stepwise increase in RSS (p-value $<0.001$ for each stepwise). Since in this study the patients were not under general 
anesthesia but only under sedation, scalp and facial muscle activity are strongly present in the EEG records, especially in the groups with $\mathrm{RSS}<=5$. This is a reason for the importance of the high frequency range in the estimation of the sedation level. This finding is according with [15], where it was established that quantitative facial surface electromyography enables discrimination of adequate vs. inadequate anesthesia, being also a useful measure of drug effect, vigilance levels and central nervous system integrity.

Hayashi et al. [10], in a research of Poincaré plot analysis in general anesthesia, indicated that the index $S D 1 / S D 2$, computed in a frequency range of $0-30 \mathrm{~Hz}$, may not be necessarily correlated with anesthesia depth in the near conscious level, and the index SD2 could estimate more accurately this light level of an anesthesia. In the present work, similarly $S D 1 / S D 2_{F R 2}$ was not able to estimate light sedation levels $(\mathrm{Pk}=0.532)$, and neither $S D 2_{F R 2}$ showed a good estimation $(\mathrm{Pk}=0.606)$. However, the index $S D 1 / S D 2_{F R 3}$ (in the range of $30-110 \mathrm{~Hz}$ ) had a $\mathrm{Pk}=0.711$, emerging as an index to be considered in the assessment of the sedation level. The individual indexes $S D 1_{F R 2}$ and $S D 2_{F R 2}$ achieve the best performance of classifying the responses in groups RSS5 and RSS6 with Acc $=67.1 \%$. In this context, $S D 1_{F R 2}$ can include the information of the relative high frequency variations of the EEG in the frequency range $0.5-30 \mathrm{~Hz}$ ( $\alpha$ and $\beta$ activity), while $S D 2$ may depend on the variations of the amplitude of the signals (shift from $\alpha$ to $\theta$ activity) [16]. It is important to note that $\delta$ activity is not strongly presented in this database where the mean BIS value was 64.4 in RSS6, the deepest sedation level.

Traditional spectral indexes as SEF95 and SpEn have not shown a high $\mathrm{Pk}$ value neither in Trial 1 (0.588 and 0.501 , respectively) nor Trial 2 ( 0.544 and 0.570 , respectively). On the other hand, BIS and CePropo had a similar behavior, being good estimators of RSS for Trial 1 but not for Trial 2, while CeRemi was a better estimator in Trial 2 than in Trial 1. High levels of remifentanil are associated with high level of analgesia, which are reflected in a reduction of the response to noxious stimuli, as it is observed in RSS6 (CeRemi $=1.45 \pm 0.728 \mathrm{ng} / \mathrm{mL}$ ). It is known that BIS is able to describe hypnotic effect as it was confirmed by results of Trial 1 , but it cannot describe the analgesic effect and the response to noxious stimulation correctly. BIS might have the same value for different concentrations of drugs and it is also possible that in low doses of analgesia a response to noxious stimuli might be observed even at low BIS values [17].

It is important to point out that no advanced techniques were applied to reject artifacts in this work, suggesting that Poincaré analysis performed well with artifacts or can be improved with a good artifact rejection. Also, the Poincaré plot has the potential to be a good approach for short-duration dynamic signals and stable long EEG signals are not required for analysis [10].

\section{CONCLUSION}

In this work, Poincaré plot was used as a nonlinear technique applied to EEG signals in order to characterize the levels of sedation-analgesia, according to observed categorical responses that were evaluated by means of Ramsay Sedation Scale (RSS). The obtained results indicated that indexes obtained from filtered EEG signal from low to high frequency range which contains EMG components improve the prediction of different stimuli responses in lightly sedated patients. Furthermore, the results showed that the ratio $S D 1 / S D 2_{F R 3}$ contains useful information about the sedation level, and $S D 1_{F R 2}$ and $S D 2_{F R 2}$ had the best performance classifying the noxious stimuli responses. Finally, it can be concluded that models including parameters from Poincaré plot emerge as a good estimator of sedation-analgesia levels.

\section{REFERENCES}

[1] I.J. Rampil, "A primer for EEG signal processing in anesthesia," Anesthesiology, 1998, 89, pp. 980-1002.

[2] H.Viertiö-Oja, V. Maja, M. Särkelä, P. Talja, N. Tenkanen, H. Tolvanen-Laakso, M. Paloheimo, A. Vakkuri, A. Yli-Hankala, P. Meriläinen, "Description of the Entropy algorithm as applied in the Datex-Ohmeda S/5 Entropy Module," Acta Anaesthesiol. Scand. 2004, 48, pp. 154-161.

[3] E.W. Jensen, P. Lindholm, S. Henneberg, “Auto Regressive Modeling with Exogenous Input of auditory evoked potentials to produce an on-line depth of anaesthesia index," Methods of Information in Medicine, 1996, 35, pp. 256-260.

[4] G. A. Mashour, B. A. Orser, M. S. Avidan, "Intraoperative awareness: neurobiology to clinical practice," Anesthesiology, 2011, 114, pp. 1218-33.

[5] V. Eichhorn, D. Henzler, M. F. Murphy, "Standardizing care and monitoring for anesthesia or procedural sedation delivered outside the operating room," Current Opinion in Anaesthesiology, 2010, 23, pp. 494-499.

[6] J. J. Pandit, T. M. Cook, "National Institute for Clinical Excellence guidance on measuring depth of anaesthesia: limitations of EEG-based technology," British Journal of Anaesthesia, 2014, 112, pp. 385-6.

[7] C. J. Chisholm, J. Zurica, D. Mironov, R. R. Sciacca, E. Ornstein, and E. J. Heyer, "Comparison of electrophysiologic monitors with clinical assessment of level of sedation.," Mayo Clinic proceedings. 2006, 81, pp. 46-52.

[8] U. Melia, M. Vallverdu, M. Jospin, E.W. Jensen, J.F. Valencia, F. Claria, P. Gambus, P. Caminal, "Prediction of nociceptive responses during sedation by time-frequency representation," IEEE-EMBS Proc, Annu Int Conf of the IEEE, 2013, pp. 2547-2550.

[9] C. K. Karmakar, A. H. Khandoker, J. Gubbi, and M. Palaniswami, "Complex correlation measure: a novel descriptor for Poincaré plot," BioMedical Engineering, 2009, pp. 1-12.

[10] K. Hayashi, T. Yamada, and T. Sawa, "Comparative study of Poincaré plot analysis using short electroencephalogram signals during anaesthesia with spectral edge frequency 95 and bispectral index," Anaesthesia, 2015, pp. 310-317.

[11] M. A. Ramsay, T. M. Savege, B. R. Simpson, R. Goodwin, "Controlled sedation with alphaxalone-alphadolone," Br. Med. J., 1974, 2, pp. 656659.

[12] A. Goshvarpour, A. Goshvarpour, S. Rahati, V. Saadatian, M. Morvarid, "Phase space in EEG signals of women refferred to meditation clinic," J. Biomedical Science and Engineering, 2011, 4, pp. 479-482

[13] J. Kortelainen and T. Seppanen, "Electroencephalogram-based depth of anaesthesia measurement: Combining opioids with hypnotics," Trends in Anaesthesia and Critical Care, 2013, pp 270-278.

[14] W. D. Smith, R. Dutton, N.T. Smith, "Measuring the performance of anesthetic depth indicators," Anesthesiology, 1996, 84, pp. 38-51.

[15] M. Paloheimo, "Quantitative surface electromyography (qEMG): applications in anaesthesiology and critical care," Acta Anaesthesiol Scand Suppl., 1990, 93, pp.1-83.

[16] K. Hayashia, N. Mukaia, T. Sawa, "Poincaré analysis of the electroencephalogram during sevoflurane anesthesia," Clinical Neurophysiology, 2015, pp. 404-411

[17] E. Brocas, H. Dupont, C. Paugam-Burtz, F. Servin, J. Mantz, J.M. Desmonts, "Bispectral index variations during tracheal suction in mechanically ventilated critically ill patients: effect of an alfentanil bolus," Intensive Care Med. 2002, 28, pp. 211-213. 